\title{
Phase transition between the cholesteric and twist grain boundary $\mathrm{C}$ phases
}

\author{
I. Luk'yanchuk ${ }^{1,2, *}$ \\ ${ }^{1}$ L.D.Landau Institute for Theoretical Physics, Moscow, Russia. \\ ${ }^{2}$ Departamento de Fisica, Universidade Federal de Minas Gerais, \\ Caixa Postal 702, 30161-970, Belo Horizonte, Minas Gerais, Brazil
}

(October 11, 2018)

\begin{abstract}
The upper critical temperature $T_{c 2}$ for the phase transition between the Cholesteric phase $\left(N^{*}\right)$ and the Twist Grain Boundary $\mathrm{C}$ phase with the layer inclination tilted to the pitch axis $\left(T G B_{C t}\right)$ in thermotropic liquid crystals is determined by the mean field Chen-Lubensky approach. We show that the $N^{*}-T G B_{C t}$ phase transition is split in two with the appearance of either the $T G B_{A}$ or the $T G B_{2 q}$ phase in a narrow temperature interval below $T_{c 2}$. The latter phase is novel in being superposed from two degenerate $T G B_{C t}$ phases with different (left and right) layers inclinations to the pitch axis.
\end{abstract}




\section{INTRODUCTION}

A Twist Grain Boundary $(T G B)$ state that appears as an intermediate state at the Cholesteric $\left(N^{*}\right)$ - Smectic $(S m)$ phase transition in chiral thermotropic liquid crystals was predicted theoretically by Renn and Lubensky [1] in 1988 and then, one year later, was independently observed experimentally [2,3]. Since that time a wealth of properties of this new state were discovered in a number of experimental 44 13 and theoretical [14 17] investigations.

The results of these studies and the results of the present paper are summarized in the phase diagram of Fig. 1 where the parameters $t, \sigma_{\perp}$ (whose meanings will be explained in Sect. II) are controlled by the following experimental conditions: temperature, concentration, pressure etc. The reason for such a variety of intermediate phases is that, the direct $N^{*}$ $S m$ transition can not occur in a continuous way since the cholesteric twist of the director, $\mathbf{n}(r)=\left(0, \sin \left(k_{0} x\right), \cos \left(k_{0} x\right)\right)$, is incompatible with the smectic layered structure. The last one in chiral liquid crystals is known to be of either $S m A$ or $S m C^{*}$ type: in $S m A$ the director $\mathbf{n}$ is parallel to the layers modulation vector $\mathbf{q}$ whereas in $S m C^{*}$ it is tilted with respect to $\mathbf{q}$ by a constant angle $\theta_{0}$ and forms a conical precession along the normal to layers. Therefore, the transition occurs either by the first order untwisting of $\mathbf{n}(r)$ or via formation of intermediate $T G B$ phases. The actual sequence of the intermediate phases depends on the final $S m$ state that occurs at low temperature. There is only one intermediate phase $\left(T G B_{A}\right)$ when the transition goes to $S m A$ [1, 14] and a series of phases when the transition goes to $S m C^{*} 15$.

The general structure of the TGB state is shown in Fig. 2a. The compromise between the cholesteric twist of $\mathbf{n}(r)$ and layered structure of $S m A, C^{*}$ is achieved by formation of a set of rotated smectic slabs (blocks), normal to those being follow the pitch $\mathbf{n}(r)$. The slabs are separated by grain boundaries consisting of a series of equally spaced screw dislocations that provide the junction of the layers in adjusting slabs. The slab width $l_{b}$, dislocation spacing $l_{d}$, director pitch $P$, and layer spacing $d$ are related by the following topological constraint [1]:

$$
2 \pi l_{b} l_{d}=P d
$$

Coupling of the director with the modulation vector $\mathbf{q}$ results in the unbending of $\mathbf{n}(r)$ close to the block center. When temperature decreases the director pitch and slab width diverge with further untwisting of $\mathbf{n}(r)$. Finally, they tend to infinity corresponding to the transition to the $S m$ state.

The variety of $T G B$ phases in Fig. 1 is provided by the different internal structure of $T G B$ blocks that are shown in Fig.2b-e. Generally, TGB blocks are reminiscent of the final $S m$ state that occurs at low temperature. Therefore, the $T G B_{A}$ block is just the $S m A$ slab, shown in Fig. 2b, confined by grain boundaries, the layers modulation vector $\mathbf{q}$ being parallel to the director $\mathbf{n}$ in the slab center [1]. Similarly, the $T G B_{C}$ slab is provided by smectic layers that are inclined to $\mathbf{n}$ by the angle $\sim \theta_{0}$. The inclination, however, can be done in other ways: when tilted layers are either parallel to the pith axis $x$ as in Fig. 2b, or tilted to it as in Fig.2c. We call these phases $T G B_{C p}$ and $T G B_{C t}$. The blocks of the $T G B_{C p}$ and $T G B_{C t}$ phases actually have the structure of slabs of differently oriented $S m C$, as distinguished from 
its chiral analog $S m C^{*}$ by the absence of the director precession. Under certain conditions a transition $S m C \rightarrow S m C^{*}$ occurs inside the $T G B_{C}$ slabs 15. The corresponding $T G B_{C^{*}}$ phase that appears close to the bulk $S m C^{*}$ phase (see Fig. 1) will be not considered in this paper.

Originally the $T G B_{C p}$ phase was assumed to be an intermediate $T G B$ state at the $N^{*}-S m C^{*}$ transition [15] (there it was called $T G B_{C}$ ). However, x-ray experiments [7] and theoretical estimations [17] demonstrate that the $T G B_{C t}$ phase is indeed more stable. In [17] this phase was called as the Melted Grain Boundary $(M G B)$ phase to stress that the smectic order parameter vanishes at grain boundaries because of the small distance between screw dislocations. We prefer, however, to use the $T G B_{C t}$ notation to emphasize the geometrical structure of this phase.

In this paper we revise the calculation of [15] for the upper critical temperature $T_{c 2}\left(M_{A^{-}}\right.$ $M_{0}-M_{1}-M_{C}$ line in Fig. 1 for the $N^{*}-T G B_{C}$ transition, taking into account the recent proof of the stability of the $T G B_{C t}$ phase [0,17] that was not considered in [15]. We confirm and expand the estimation of [15] to the whole region of parameters. In addition we calculate the principal parameters of the $T G B_{C t}$ phase: upper critical temperature, slab width $l_{b}$ and the X-ray diffraction pattern that can be measured experimentally.

Several features that modify the phase diagram calculated in [16, 15 follow from our analysis. The $T G B_{A}$ phase that was shown in [1,14 to be stable when the transition is to the $S m A$ phase, penetrates also into the $S m C^{*}$ region as a narrow stripe in between the $N^{*}$ and $T G B_{C t}$ phases, and finishes in the tetracritical point $M_{0}$ far inside this region. The $N^{*}-T G B_{C t}$ transition is split either by this $T G B_{A}$ stripe or by the narrow region of the new $T G B_{2 q}$ phase. The $T G B_{2 q}$ slab is superposed from two equivalent $S m C$ populations with left and right layers inclined to the pitch axis as shown in Fig. 2e.

This new phase can be viewed as a kind of standing density wave quantized by the grain boundaries. In reality it can be observed in between the $N^{*}-T G B_{A}-T G B_{C t^{-}} T G B_{2 q}$ tetracritical point $M_{0}$ and the point $M_{1}$. Location of these points is calculated in this paper. The upper critical temperature $T_{c 2}$ should have a kink at $M_{0}$. Under certain conditions the enhancement (oscillation) of $T_{c 2}$ between $M_{0}$ and $M_{1}$ can be observed.

A remarkable feature of the $T G B_{A}$ phase was noted in [1] to be an analogy with the Abrikosov vortex state in superconductors in a magnetic field. This completed an analogy between the superconducting transition in metals and the phase transition between Nematic $(N)$ and $S m A$ phases in liquid crystals, first pointed by de Gennes [18,19. In the present article we show that the $T G B_{C t}$ phase is the analog of the mixed state in superconductors with a space-modulated order parameter (like Larkin-Ovchinnikov-Fulde-Ferrel phases [20.21]), providing that modulation is perpendicular to the magnetic field. Another interesting analogy we discuss is the similarity between the $T G B_{A}-T G B_{C t}$ transition and the transition between the symmetry differing phases in an "unconventional" superconductor $U \mathrm{Pt}_{3}$ in the magnetic field [22].

\section{BASIC EQUATIONS}




\section{A. The chiral Chen-Lubensky Model}

On a quantitative level, the appearance of $T G B$ state is described by the Chen-Lubensky (CL) model [23], which is known to be a quite general approach in explaining various phase transitions between cholesteric (nematic) phases and modulated smectic phases. In this model the cholesteric and smectic phases are described by two coupled order parameters: by the twisted director $n(r)$ and the space modulated complex function $\psi(r)$, where the modulation of the smectic density is given by the real part of $\psi(r)$. The resulting energy consists of two parts:

$$
\mathcal{F}_{C L}=\mathcal{F}_{\psi}+\mathcal{F}_{F}
$$

where the elastic Frank energy:

$$
\begin{aligned}
\mathcal{F}_{F}=\frac{1}{2} & K_{1}(\operatorname{div} \mathbf{n})^{2}+\frac{1}{2} K_{2}\left(\mathbf{n} \cdot \operatorname{curl} \mathbf{n}-k_{0}\right)^{2} \\
& +\frac{1}{2} K_{3}(\mathbf{n} \times \operatorname{curl} \mathbf{n})^{2}
\end{aligned}
$$

provides the twisted texture of the director. In the cholesteric phase $\mathbf{n}(r)=$ $\left(0, \sin \left(k_{0} x\right), \cos \left(k_{0} x\right)\right)$. The chirality is provided by the parameter $k_{0}$. When $k_{0}=0$, expression (3) reduces to the elastic energy of the nematic phase with $\mathbf{n}=$ const.

The smectic state is described by the Ginzburg-Landau (GL) functional $\mathcal{F}_{\psi}$, which has a finite- $q$ instability for the order parameter $\psi(r)$ provided by a gauge derivative $\mathbf{D}=\nabla-i q_{0} \mathbf{n}$ :

$$
\begin{aligned}
\mathcal{F}_{\psi}= & a\left(T-T_{N A}\right)|\psi|^{2}+\frac{1}{2} g|\psi|^{4}+\left(C_{\|} n_{i} n_{j}\right. \\
& \left.+C_{\perp}\left(\delta_{i j}-n_{i} n_{j}\right)\right)\left(\mathbf{D}_{i} \psi\right)\left(\mathbf{D}_{\jmath} \psi\right)^{*}+D\left(\mathbf{D}^{2} \psi\right)\left(\mathbf{D}^{2} \psi\right)^{*}
\end{aligned}
$$

We take the quartic in the gradient term in the isotropic form $D\left(\mathbf{D}^{2} \psi\right)\left(\mathbf{D}^{2} \psi\right)^{*}$ which is slightly different from the original CL model where this term was written as: $D_{\perp}\left(\delta_{i j}-n_{i} n_{j}\right)\left(\delta_{k l}-n_{k} n_{l}\right)\left(\mathbf{D}_{i} \mathbf{D}_{j} \psi\right)\left(\mathbf{D}_{k} \mathbf{D} \psi\right)^{*}$. This does not change the final results but simplifies the calculations.

Now we give a brief review of the properties of the CL model. In the nonchiral case (when $k_{0}=0$ ), a second order phase transition from nematic to smectic phases takes place [23]. The type of the smectic phase depends on the sign of $C_{\perp}$ (terms with $C_{\|}$and $D$ are assumed to be positive). When $C_{\perp}>0$, a transition to the $S m A$ phase with the order parameter $\psi(r) \sim \psi_{0} e^{i q_{0} \mathbf{n r}}$ occurs at $T=T_{N A}$. When $C_{\perp}<0$ an additional transversal to $\mathbf{n}$ modulation occurs, resulting in a $S m C$ order parameter $\psi(r) \sim \psi_{0} e^{i \mathbf{q r}}$ with $\mathbf{q}=\left(q_{0} \mathbf{n}, \mathbf{q}_{C}\right)$. This modulation is stabilized by the quartic gradient term at wave vector $q_{C}=\left(-C_{\perp} / 2 D\right)^{1 / 2}$ which gives the layers inclination angle as $\theta_{0}=\arctan \left(q_{C} / q_{0}\right)=\arctan \left(-C_{\perp} / 2 D q_{0}^{2}\right)^{1 / 2}$. The $N-S m C$ transition occurs at $T_{N C}=T_{N A}+C_{\perp}^{2} / 4 D$.

In a chiral case [14] (when $k_{0} \neq 0$ ), the situation is more complicated since the gauge derivatives and anisotropic coefficients in the gradient terms in Eq.(1) depend on the direction of $\mathbf{n}$ which is not uniform in a space. This provides a coupling of the order parameters $\mathbf{n}(r)$ and $\psi(r)$ leading to an untwisting of $\mathbf{n}(r)$ when the smectic layers are formed. Such 
a process occurs either directly, by the first order transition, or via the intermediate $T G B$ state. In both cases the final smectic phases are again either $S m A$ or $S m C$.

The scenario of the phase transition depends on the parameters of the CL model. We formulate those conditions below. When the direct $N^{*}-S m A, C^{*}$ transition takes place, the critical temperatures are calculated by comparison of the energies of the $N^{*}$ and $S m A, C^{*}$ phases [14]. The $N^{*}-S m A-S m C^{*}$ tricritical point in the $\left(T, C_{\perp}\right)$ plane was found to be:

$$
\left(T^{*}, C_{\perp}^{*}\right)=\left(T_{N A}-\left(g K_{2}\right)^{1 / 2} k_{0} / a,\left(g K_{2}^{3}\right)^{1 / 2} k_{0} / 2 a K_{3} q_{0}^{2}\right)
$$

The transition lines are given by:

$$
\begin{aligned}
T_{N^{*} A} & =T^{*}, C_{\perp}>C_{\perp}^{*} \\
T_{A C^{*}} & =T_{N A}+\left(T_{N^{*} A}-T_{N A}\right) \cdot C_{\perp}^{*} / C_{\perp}, C_{\perp}<C_{\perp}^{*} \\
T_{N^{*} C^{*}} & =T_{N^{*} A}+\left(C_{\perp}-C_{\perp}^{*}\right)^{2} / 4 a D, C_{\perp}<C_{\perp}^{*}
\end{aligned}
$$

When the transition occurs via an intermediate $T G B$ state, the critical temperatures and the detailed structure of the $T G B$ state are provided by a nonuniform solution of the GL equation obtained from (4):

$$
\begin{aligned}
& a\left(T-T_{N A}-\frac{C_{\perp}^{2}}{4 D}\right) \psi+g|\psi|^{2} \psi \\
= & \left(C_{\|}-C_{\perp}\right)(\mathbf{n D})^{2} \psi-D\left(-\mathbf{D}^{2}+\frac{C_{\perp}}{2 D}\right)^{2} \psi
\end{aligned}
$$

where, $\widehat{A}^{2} \psi=\widehat{A}(\widehat{A} \psi)$.

In this paper we are interested in the structure of the $T G B$ state just below the $N^{*}-T G B$ phase transition that takes place at the upper critical temperature $T_{c 2}$. We consider the case when $C_{\perp}<0$, that is when the system has a tendency to form the $S m C^{*}$ phase at low temperature. In the following subsection we derive the basic equations that describe this transition.

\section{B. Cholesteric-TGB transition}

The order parameter in the $T G B$ state below $T_{c 2}$ is given by a periodic superposition of rotating blocks equally shifted by a distance $l_{b}$

$$
\psi(r)=\sum_{m} e^{i \gamma_{m}} f\left(x+m l_{b}\right) e^{i \mathbf{q}_{\perp m} \mathbf{r}}
$$

The component of the vector transverse to the pitch $\mathbf{q}_{\perp m}=q_{0}\left(0, \sin \left(k_{0} m l_{b}+\varphi\right), \cos \left(k_{0} m l_{b}+\varphi\right)\right)$, follows the director twist: $\mathbf{n}(r)=\left(0, \sin \left(k_{0} x\right), \cos \left(k_{0} x\right)\right)$ with phase advance (retardation) $\varphi$. Factors $e^{i \gamma_{m}}$ are the degenerate phason degrees of freedom [1].

The block profile function $f(x)$ is localized within the block width $l_{b}$ and, together with the phase $\varphi$, provides the structure of the $T G B$ slab. In the $T G B_{A}$ phase $\varphi=0$ and $f(x)$ is a centered bell-shape function (which has a Gaussian profile $\exp \left(-k_{0} x^{2} / 2 q_{0}\right)$ at $C_{\perp} \gg 0$ 证). 
The structure of the $T G B_{C p}$ slab (Fig. 2c) is given by a centered bell-shape function $f(\bar{x})$ and nonzero advancing (retarding) phase angle $\varphi \simeq \theta_{0}$ [15 that results in the tilting of the layers parallel to the pitch axis. In the $T G B_{C t}$ phase the angle $\varphi$ is equal to zero and $f(x)$ is modulated in the $x$ direction as $\exp ( \pm i \theta x)$. This corresponds to the right (left) transverse inclination of the layers as shown in Fig. 2d.

The profile function $f(x)$ of the slab, located at the origin, and phase $\varphi$ are found by solving of Eq.(17) with the substitution $\psi(r)=f(x) e^{i \mathbf{q}_{\perp 0} \mathbf{r}} \simeq f(x) e^{i q_{0}(z+\varphi y)}$.

Several simplifications are used in Renn-Lubensky theory. Usually the block width $l_{b}$ is much smaller than the cholesteric pitch $P=2 \pi / k_{0}$. Therefore, on the scale of $l_{b}$ the twist of $\mathbf{n}(r)$ is minimal and approximately is written as $\mathbf{n}(r) \approx\left(0, k_{0} x, 1-\left(k_{0} x\right)^{2} / 2\right)$. Next, just below $T_{c 2}$ the amplitude of $f(x)$ is small and only the terms linear in $f(x)$ are relevant in Eq.([).

The corresponding linearized equation for $f(x)$ and $\varphi$ is:

$$
\begin{aligned}
a(T- & \left.T_{N A}-C_{\perp}^{2} / 4 D\right) f=-\mathcal{H} f \quad \text { where } \\
\mathcal{H}= & D\left(-\partial_{x}^{2}+\left(q_{0} k_{0}\right)^{2}\left(x-\varphi / k_{0}\right)^{2}+\frac{C_{\perp}}{2 D}\right)^{2} \\
& +\left(C_{\|}-C_{\perp}\right) \frac{q_{0}^{2} k_{0}^{4}}{4}\left(x^{2}-2 x \varphi / k_{0}\right)^{2}
\end{aligned}
$$

This equation has a set of localized eigenstates $f_{n}(x)$ with a discrete spectrum of eigentemperatures $T_{n}$. The upper critical temperature $T_{c 2}$ of the $N^{*}-T G B$ transition is provided by the maximal value of $T_{n}$. The block profile function is given by the corresponding eigenfunction $f_{n c 2}(x)$.

We assume further that $\varphi=0$, that is, the parallel layers' inclination according to [17] does not occur. The justification of this approximation will be presented in Sect. IIID.

It is convenient to use the dimensionless units:

$$
\begin{gathered}
\bar{x}=q_{0} x, \bar{\partial}_{x}=q_{0}^{-1} \partial_{x}, \sigma_{\|}=\frac{C_{\|}}{4 D q_{0}^{2}}, \sigma_{\perp}=-\frac{C_{\perp}}{2 D q_{0}^{2}}, \\
t=a\left(T-T_{N A}\right) / D q_{0}^{4}, \quad b=k_{0} / q_{0}, \overline{\mathcal{H}}=\mathcal{H} / D q_{0}^{4}
\end{gathered}
$$

We discuss first the values of parameters $b, \sigma_{\perp}, \sigma_{\|}$. Parameter $b$ has a sense of the power of the cholesteric twist. Usually in chiral liquid crystals the interlayer smectic spacing is much smaller than cholesteric pitch having $b \ll 1$. The small tilting angle $\theta_{0}$ results in $\sigma_{\perp}=\tan ^{2} \theta_{0} \ll 1$. In contrast, parameter $\sigma_{\|}$, which is related to the ratio of the layer compression elastic constant $C_{\|} q_{0}^{2}$ to the layer curvature energy $D q_{0}^{4}$, is larger than one [17.24] and therefore is much large than $\sigma_{\perp}$.

Neglecting $C_{\perp}$ in comparison with $C_{\|}$in the last term of Eq.(9), we rewrite it in dimensionless units as:

$$
\begin{aligned}
& \left(t-\sigma_{\perp}^{2}\right) f=-\overline{\mathcal{H}} f \quad \text { where } \\
& \overline{\mathcal{H}}=\left(-\bar{\partial}_{x}^{2}+b^{2} \bar{x}^{2}-\sigma_{\perp}\right)^{2}+\sigma_{\|} b^{4} \bar{x}^{4}
\end{aligned}
$$


The order parameter $\psi(r)$ of the $T G B$ state is reproduced by substitution of corresponding eigenfunction $f(x)$ into (8). Then, one can calculate the free energy $\mathcal{F}_{C L}(\mathbb{8})$ of the $T G B$ state as a function of the slab width $l_{b}$ or, more conveniently, as a function of the geometrical factor $l_{b} / l_{d}$. The actual value of this ratio is found by minimization of $\mathcal{F}_{C L}$ with respect to $l_{b} / l_{d}$. It was found [1] that $l_{b} / l_{d} \simeq 0.9 \varepsilon$ when $C_{\perp} \gg 0$ and the $T G B_{A}$ phase has a Gaussian profile in the slab. The factor $\varepsilon$ depends the relative strengths of cholesteric splay (bend) and the twist elastic energies $K_{1,3} / K_{2}$. It varies from $\varepsilon=0$ when $K_{1,3} / K_{2}=0$ (the calculation in this case is reduced to minimizating the Abrikosov factor $\left.\beta=\left\langle\psi^{4}\right\rangle /\left\langle\psi^{2}\right\rangle^{2}\right)$, to $\varepsilon \sim 1.5$ when $K_{1,3} / K_{2}$ is large. We will use this result to evaluate $l_{b} / l_{d}$ in the $T G B_{A, C t}$ phases when $C_{\perp}<0$.

\section{RESULTS}

\section{A. General}

In this section we calculate the eigenstates of Eq.(11) that correspond to the upper critical temperature $t_{c 2}$, and discuss the block structure just below $t_{c 2}$ for the different types of $T G B$ state at $\sigma_{\perp}>0$.

Note that eigentemperatures $t_{n}$ (including $t_{c 2}$ ) and eigenfunctions $f_{n}(x)$ are generally functions of $b, \sigma_{\perp}, \sigma_{\|}$. Because of the scaling properties resulting from Eq.(11):

$$
\begin{aligned}
t_{n}\left(b, \sigma_{\|}, \sigma_{\perp}\right) & =\sigma_{\perp}^{2} \cdot t_{n}\left(b / \sigma_{\perp}, \sigma_{\|}, 1\right), \\
f_{n}\left(\bar{x}, b, \sigma_{\|}, \sigma_{\perp}\right) & =f_{n}\left(\sigma_{\perp}^{1 / 2} \bar{x}, b / \sigma_{\perp}, \sigma_{\|}, 1\right) .
\end{aligned}
$$

parameter $\sigma_{\perp}$ can be excluded if one considers the dependence $t_{n}(b)$ on the rescaled coordinates $b / \sigma_{\perp}, t / \sigma_{\perp}^{2}$.

To proceede with the diagonalization of Eq.(11) for $\sigma_{\perp}>0$, recall first the results of [1].14] for the opposite case of $\sigma_{\perp}<0$ when a transition occurs to the $T G B_{A}$ phase. When $\left|\sigma_{\perp}\right|$ is large enough, the operator $\overline{\mathcal{H}}$ was shown [1] to be truncatable to the more simple form of the harmonic oscillator $-2 \sigma_{\perp}\left(-\bar{\partial}_{x}^{2}+b^{2} \bar{x}^{2}\right)+\sigma_{\perp}^{2}$. The lowest eigenstate gives the Gaussian profile $e^{-b \bar{x}^{2}}$ of the $T G B_{A}$ block and the upper critical temperature $t_{c 2}=2 \sigma_{\perp} b$. This result can be improved if one considers the residual part of $\overline{\mathcal{H}}$ as a perturbation:

$$
t_{c 2}=2 \sigma_{\perp} b-\left(1+3 \sigma_{\|} / 4\right) b^{2}
$$

The second term is smaller than the first one if $-\sigma_{\perp} / b>0.5+0.38 \sigma_{\|}$, which is the condition of applicability of the approximation. The opposite case of $\sigma_{\perp} / b \sim 0$ will be discussed later.

Figure 3 shows the result of numerical diagonalization of (11) for different $\sigma_{\|}$when $\sigma_{\perp}>0$. We use the $t / \sigma_{\perp}^{2}, b / \sigma_{\perp}$ coordinates to trace the two highest eigentemperatures (the maximal one corresponds to $t_{c 2}$ ) as function of $b$. Note first the concurrence of two eigenstates with close eigentemperatures $t_{+}$and $t_{-}$that give the upper critical temperature $t_{c 2}=\max \left(t_{+}, t_{-}\right)$. These levels often cross when $b$ changes, resulting in the oscillating behavior of $t_{c 2}(b)$. The oscillations of $t_{c 2}(b)$ are strong at $\sigma_{\|}=0$ and more pronounced still at large values of $\sigma_{\|}$.

The appearance of these nearly degenerate eigenstates is related to two equivalent, right and left inclinations of layers in the $T G B_{C t}$ slab. The corresponding block profile functions, 
$f_{R}(\bar{x})$ and $f_{L}(\bar{x})$, however, can not be the eigenfunctions of Eq.(11) since they do not possess the definite parity with respect to the symmetry operation $x \rightarrow-x$, which is a property of $\overline{\mathcal{H}}$. The proper eigenstates $f_{ \pm}$with eigentemperatures $t_{ \pm}$are constructed as superpositions of both populations as $f_{ \pm}=f_{R} \pm f_{L}$ that results in the $T G B_{2 q}$ phase just below $t_{c 2}$. The function $f_{ \pm}(x)$ has cos- (sin- ) like oscillations and can be viewed as a standing wave between the grain boundaries. The effects of commensurability of this wave with block width $l_{b}$ stabilize either the even cos-like or the odd sin-like behavior as the lowest state to have a vanishing order parameter at the grain boundaries. The block width $l_{b}$ changes as a function of $b$ that alternates the order of the $f_{ \pm}$eigenstates leading to the oscillations in $t_{c 2}(b)$.

The tendency to $S m C$ slab formation at lower temperatures results in the further transition from the $T G B_{2 q}$ to $T G B_{C t}$ phase determined by the lowest from $t_{+}, t_{-}$critical temperatures renormalized by the nonlinear term $g|\psi|^{2} \psi$ in Eq.(7). Below this second transition, one of the populations starts to be suppressed and a block profile function is constructed now from both the $f_{+}$and $f_{-}$eigenstates to form the $T G B_{C p}$ phase with either a $f_{L}$ or $f_{R}$ profile of the slab.

Therefore, we conclude that the $N^{*}-T G B_{C t}$ transition is always split by the intermediate $T G B_{2 q}$ phase. At high $b$ the period of oscillations of $f_{ \pm}(x)$ becomes larger than its localization length, and the lowest eigenstate $f_{+}(x)$ has the single-peak profile of the $T G B_{A}$ phase. The $N^{*}-T G B_{A^{-}}-T G B_{C t}-T G B_{2 q}$ tetracritical point $M_{0}$ corresponds therefore to the highest in $b$ intersection of $t_{ \pm}$eigentemperatures. As follows from Fig. 3, the appearance of the $T G B_{2 q}$ phase becomes practically invisible when $b$ decreases below the second intersection of $t_{ \pm}$at the point $M_{1}$. We expect, therefore, that only the odd $T G B_{2 q}$ phase between $M_{0}$ and $M_{1}$ can be observed in reality.

Equation (11) can be solved analytically in the two limit cases $\sigma_{\|}=0$ and $\sigma_{\|} \gg 1$, when the operator $\overline{\mathcal{H}}$ can be truncated to a more simple form. Although the case $\sigma_{\|}=0$ does not correspond to the real situation of $\sigma_{\|}>1$ we consider it first since it clarifies the qualitative structure of the phase diagram that conserves also at large $\sigma_{\|}$.

\section{B. Case $\sigma_{\|}=0$}

When $\sigma_{\|}=0$, operator $\overline{\mathcal{H}}$ is a polynomial Schrödinger operator for the harmonic oscillator:

$$
\overline{\mathcal{H}}=\sigma_{\perp}^{2}-2 \sigma_{\perp}\left(-\bar{\partial}_{x}^{2}+b^{2} \bar{x}^{2}\right)+\left(-\bar{\partial}_{x}^{2}+b^{2} \bar{x}^{2}\right)^{2}
$$

Therefore, it has the same set of oscillator eigenfunctions:

$$
f_{n}(\bar{x})=H_{n}(\sqrt{b} \bar{x}) e^{-\bar{x}^{2} b / 2}
$$

where $H_{n}$ are the Hermitian polynomials. The corresponding eigentemperatures are given by the equation:

$$
t_{n}=2 \sigma_{\perp}(2 n+1) b-(2 n+1)^{2} b^{2}
$$

The oscillations of $t_{c 2}(b)=\max t_{n}(b)$ (Fig.3a) are related to the quantum number $n_{c 2}$ of the lowest eigenstate that changes with $b$, unlike what occurs in the harmonic oscillator where the $n=0$ eigenlevel is always the lowest one. 
When $b>\sigma_{\perp} / 2$, the $n=0$ eigenlevel does correspond to the upper critical temperature $t_{c 2}=2 \sigma_{\perp} b-b^{2}$. The block profile function has a Gaussian shape $e^{-\bar{x}^{2} b / 2}$ in the $T G B_{A}$ phase. The slab width is calculated as in [1]:

$$
\bar{l}_{b}=2.2 \varepsilon^{1 / 2} / b^{1 / 2}
$$

When $b<\sigma_{\perp} / 2, n_{c 2}$ is given by $\sigma_{\perp} / 2 b-1 / 2$ rounded to the nearest integer. The oscillating upper critical temperature $t_{c 2}(b)$ tends to $\sigma_{\perp}^{2}$ when $b$ vanishes as shown in Fig. 3a. The slab width is given by the width of the polynomial $H_{n c 2}(\sqrt{b} \bar{x})$ as:

$$
\bar{l}_{b}=\sigma_{\perp}^{1 / 2} / b
$$

The block profile function $f_{n c 2}(\bar{x})$ oscillates as $\cos \left(\lambda \bar{x}+\pi n_{c 2}\right)$ with period $\lambda=2 \pi /\left(2 n_{c 2} b\right)^{1 / 2}$ $\simeq 2 \pi / \sigma_{\perp}^{1 / 2}$. This corresponds to the slab of the $T G B_{2 q}$ phase which is the superposition of two $S m C$ slabs with layers inclined at $\theta_{0}= \pm \sigma_{\perp}^{1 / 2}$. The parity of $f_{n}(\bar{x})$ alternates with $n$ resulting in the different parity of $f_{n_{c 2}}$ and $f_{n_{c 2} \pm 1}$ eigenstates. So, the admixture of $i f_{n_{c 2} \pm 1}$ eigenfunctions with the $T G B_{2 q}$ profile function $f_{n_{c 2}}$ that occurs at some critical temperature below $t_{c 2}$ corresponds to the $T G B_{2 q}-T G B_{C t}$ transition.

The tetracritical point $M_{0}$, where $N^{*}-T G B_{A^{-}} T G B_{C t^{-}} T G B_{C t}$ phases meet is given by $b_{0}=$ $\sigma_{\perp} / 2, t_{0}=3 \sigma_{\perp}^{2} / 4$

\section{Case $\sigma_{\|} \gg 1$}

In the limit $\sigma_{\|} \gg 1$ one can neglect $b^{2} \bar{x}^{2}$ in (11) and rewrite $\overline{\mathcal{H}}$ as:

$$
\overline{\mathcal{H}}=\left(\bar{\partial}_{x}^{2}+\sigma_{\perp}\right)^{2}+\sigma_{\|} b^{4} \bar{x}^{4}
$$

The eigentemperatures and eigenfunctions of Eq.(19) have in additional to (12) the scaling properties:

$$
\begin{aligned}
t_{n}\left(b, \sigma_{\|}, \sigma_{\perp}\right) & =\sigma_{\perp}^{2} \cdot t_{c 2}\left(b \sigma_{\|}^{1 / 4} / \sigma_{\perp}, 1,1\right), \\
f_{n}\left(\bar{x}, b, \sigma_{\|}, \sigma_{\perp}\right) & =f_{n}\left(\sigma_{\perp}^{1 / 2} \bar{x}, b \sigma_{\|}^{1 / 4} / \sigma_{\perp}, 1,1\right) .
\end{aligned}
$$

So, $t_{c 2}\left(b, \sigma_{\|}, \sigma_{\perp}\right)$ at large $\sigma_{\|}$is defined by the universal function $t_{c 2}(b, 1,1)$. We therefore use the scaled coordinates $b \sigma_{\|}^{1 / 4} / \sigma_{\perp}, t / \sigma_{\perp}^{2}$ for large $\sigma_{\|}$to trace $t_{c 2}(b)$ that is obtained from numerical diagonalization of (19) (Fig. 3c).

An important conclusion that followes from Fig. $3 \mathrm{c}$ is that the phase diagram for large $\sigma_{\|}$ posseses the same features as for $\sigma_{\|}=0$. It includes the domains of the $T G B_{A}$ and $T G B_{2 q}$ phases that precede the transition to the $T G B_{C t}$ phase. The $T G B_{A}$ phase exists above the tetracritical point $M_{0}$ and the $T G B_{2 q}$ phase is important in between the points $M_{0}$ and $M_{1}$. From Fig. 3c one locates the points $M_{0}, M_{1}$ at $b \sigma_{\|}^{1 / 4} / \sigma_{\perp} \simeq 0.36$ and $b \sigma_{\|}^{1 / 4} / \sigma_{\perp} \simeq 0.19$.

Consider now the transition to the $T G B_{C}$ phase at low field $b \ll 0.36 \sigma_{\perp} / \sigma_{\|}^{1 / 4}$ (i.e., below $M_{0}$ ) and to the $T G B_{A}$ phase at high field $b \gg 0.36 \sigma_{\perp} / \sigma_{\|}^{1 / 4}$ (i.e., above $M_{0}$ ). Both the asymptotics for $t_{c 2}(b)$ are shown in Fig. 3c by the dashed lines. 
Low fields: $b \ll 0.36 \sigma_{\perp} / \sigma_{\|}^{1 / 4}$

At low field when the block width $\bar{l}_{b}$ diverges, one can assume that blocks have the structure of $S m C$ slabs where the inclination of layers to the pitch axis is equal to its bulk value $\theta_{0}= \pm \arctan \sigma_{\perp}^{1 / 2}$. Then the block's profile function $f(\bar{x})$ has a high-frequency modulation $e^{ \pm i \sigma_{\perp}^{1 / 2} \bar{x}}$ where + and - signs correspond to right and left tilting respectively. This factor can be excluded by shift: $\bar{\partial}_{x} \rightarrow \bar{\partial}_{x} \pm i \sigma_{\perp}^{1 / 2}$ in Eq.(19). Next, we neglect $\bar{\partial}_{x}^{2}$ in comparison with $2 \sigma_{\perp}^{1 / 2} \bar{\partial}_{x}$ (justification of this approximation will be given below) and write $\overline{\mathcal{H}}$ as:

$$
\overline{\mathcal{H}}=-\left(2 \sigma_{\perp}^{1 / 2} \bar{\partial}_{x}\right)^{2}+\sigma_{\|} b^{4} \bar{x}^{4}
$$

Rescaling $\bar{x}$ as: $\zeta=\left(b^{4} \sigma_{\|} / 4 \sigma_{\perp}\right)^{1 / 6} \bar{x}$ simplifies (21) to an unharmonic oscillator operator:

$$
\overline{\mathcal{H}}=\left(16 \sigma_{\perp}^{2} \sigma_{\|}\right)^{1 / 3} b^{4 / 3}\left(-\partial_{\zeta}^{2}+\zeta^{4}\right)
$$

Finally, $t_{c 2}(b)$ and the corresponding eigenfunction of (21) are given by:

$$
\begin{aligned}
t_{c 2} & =\sigma_{\perp}^{2}-1.06 \cdot\left(16 \sigma_{\perp}^{2} \sigma_{\|}\right)^{1 / 3} b^{4 / 3} \\
f_{c 2}(\bar{x}) & =e^{ \pm i \sigma_{\perp}^{1 / 2} \bar{x}} \cdot g_{1}\left(\left(b^{4} \sigma_{\|} / 4 \sigma_{\perp}\right)^{1 / 6} \bar{x}\right)
\end{aligned}
$$

where 1.06 and $g_{1}(\zeta)$ are the lowest eigenvalue and eigenfunction of the unharmonic oscillator equation: $\left(-\partial_{\zeta}^{2}+\zeta^{4}\right) g_{1}=1.06 g_{1}$. The block width $\bar{l}_{b}$ is estimated as the characteristic width $\sim\left(4 \sigma_{\perp} / b^{4} \sigma_{\|}\right)^{1 / 6}$ of the function $g_{1}$. More accurately, we can calculate $\bar{l}_{b}$ in the same way as was done in [1] for the $T G B_{A}$ phase, using the suitable Gaussian approximation for $g_{1}(\zeta)$ as $e^{-0.4 \zeta^{2}}$. Finally we get:

$$
\bar{l}_{b}=3.2 \varepsilon^{1 / 2}\left(\sigma_{\perp} / b^{4} \sigma_{\|}\right)^{1 / 6}
$$

At first glance, the eigenstate (23) is doubly degenerate with respect to a sign change in the modulation phase $e^{ \pm i \sigma_{\perp}^{1 / 2}} \bar{x}$. However, this is an artifact of the approximation: the term $\bar{\partial}_{x}^{2}$ we are neglecting lifts the degeneracy and splits the transition onto two. In particular, in between the points $M_{0}$ and $M_{1}$ (Fig3c) $\bar{\partial}_{x}^{2}$ favors the odd $T G B_{2 q}$ phase given by the linear combination $\left(e^{i \sigma_{\perp}^{1 / 2} \bar{x}}-e^{-i \sigma_{\perp}^{1 / 2} \bar{x}}\right) \cdot g_{1}=2 i g_{1} \sin \sigma_{\perp}^{1 / 2} \bar{x}$. The $T G B_{C t}$ profile appears by means of an additional transition when the even combination $\sim 2 i g_{1} \cos \sigma_{\perp}^{1 / 2} \bar{x}$ admixes.

This effect, however, is as tiny (and negligible below $M_{1}$ ) as $\left\langle\bar{\partial}_{x}^{2}\right\rangle \simeq 1 / \bar{l}_{b}^{2}$ is smaller than $\left\langle 2 \sigma_{\perp}^{1 / 2} \bar{\partial}_{x}\right\rangle \simeq 2 \sigma_{\perp}^{1 / 2} / \bar{l}_{b}$, where $\bar{l}_{b}$ is given by Eq. (24) and $b \ll 0.36 \sigma_{\perp} / \sigma_{\|}^{1 / 4}$. The above estimation confirms the selfconsistency of the approximation we made. Condition $b \ll 0.36 \sigma_{\perp} / \sigma_{\|}^{1 / 4}$ is equivalent to the requirement that the wavelength of the transversal modulation $\lambda \sim \sigma_{\perp}^{-1 / 2}$ is smaller than the block width $\bar{l}_{b}$ given by (24). This clarifies the physical meaning of the approximation.

High fields: $b \gg 0.36 \sigma_{\perp} / \sigma_{\|}^{1 / 4}$

Neglecting now $\sigma_{\perp}$ in comparison with $\bar{\partial}_{x}^{2}$ we simplify $\overline{\mathcal{H}}$ as: 


$$
\overline{\mathcal{H}} \simeq \bar{\partial}_{x}^{4}+\sigma_{\|} b^{4} \bar{x}^{4}
$$

Then $t_{c 2}$ and the corresponding eigenfunction are given by:

$$
t_{c 2} \simeq-1.39 \cdot \sigma_{\|}^{1 / 2} b^{2} \quad f_{c 2}(\bar{x})=g_{2}\left(\sigma_{\|}^{1 / 8} b^{1 / 2} \bar{x}\right)
$$

where 1.39 and $g_{2}(\eta)$ are the lowest eigenvalue and eigenfunction of the operator $\partial_{\eta}^{4}+\eta^{4}$. Unlike the low field case, the eigenstate (25) is nondegenerate and described by the even bell-shaped function $g_{2}$, the lowest odd eigenstate being well separated from $g_{2}$ as shown in Fig. 3c.

The eigenfunction $g_{2}\left(\sigma_{\|}^{1 / 8} b^{1 / 2} \bar{x}\right)$ gives the $T G B_{A}$ - like profile of the block. The block width $\bar{l}_{b}$ is calculated using the results of [1] by noting that $g_{2}(\eta) \sim e^{-0.55 \eta^{2}}$ :

$$
\bar{l}_{b}=2.1 \varepsilon^{1 / 2} / \sigma_{\|}^{1 / 8} b^{1 / 2}
$$

The approximation we made is self-consistent since $\left\langle\bar{\partial}_{x}^{2}\right\rangle \simeq 1 / \bar{l}_{b}^{2}$ is indeed greater than $\sigma_{\perp}$ when $b \gg 0.36 \sigma_{\perp} / \sigma_{\|}^{1 / 4}$. Considering the truncated term $\widehat{h}=2 \sigma_{\perp} \bar{\partial}_{x}^{2}+\sigma_{\perp}^{2}$ as a perturbation, one improves the asymptotic for $t_{c 2}(b)$. The correction to $t_{c 2}$ is given by $\left\langle g_{2}|\widehat{h}| g_{2}\right\rangle \simeq$ $2 \gamma \sigma_{\|}^{1 / 4} \sigma_{\perp} b+\sigma_{\perp}^{2}$ where $\gamma=\left\langle g_{2}\left|\partial_{\eta}^{2}\right| g_{2}\right\rangle \simeq 0.5$. Finally, we get:

$$
\begin{aligned}
t_{c 2} & \simeq \sigma_{\|}^{1 / 4} \sigma_{\perp} b-1.39 \sigma_{\|}^{1 / 2} b^{2} \\
& =-1.39 \cdot\left(\sigma_{\|}^{1 / 4} b-0.36 \sigma_{\perp}\right)^{2}+0.18 \sigma_{\perp}^{2}
\end{aligned}
$$

In fact, the asymptotic (28) is valid also in the region of small negative $\sigma_{\perp} / b$. It makes the analogous expressions in [14,15] more precise and explicitly determines all the coefficients.

\section{Stability of the $T G B_{C t}$ state.}

Having calculated the transition temperature and structure of the $T G B_{C t}$ phase we discuss now its stability, namely, whether this phase has the highest upper critical temperature $t_{c 2}$ (or, alternatively, the highest upper critical field, $b_{c 2}$ ) among other $T G B$ states and under which condition a transition from $N^{*}$ to $S m C^{*}$ occurs via the intermediate $T G B_{C t}$ phase rather than via the direct first order transition.

To answer the first question, consider the competing $T G B_{C t}$ and $T G B_{C p}$ phases that can be formed at the $N^{*}-S m C^{*}$ transition. Dozov's estimation of the ratio of their upper critical fields [17], gives the factor $2\left(C_{\|} / 2 D q_{0}^{2}\right)^{1 / 4}=2\left(2 \sigma_{\|}\right)^{1 / 4}$ which is larger than one as long as $\sigma_{\|}>1$. Therefore, $b_{c 2}\left(T G B_{C t}\right)>b_{c 2}\left(T G B_{C p}\right)$ and the $T G B_{C t}$ phase is indeed more preferable.

Our calculations are in agreement with Dozov's estimation. To show this, compare $t_{c 2}\left(T G B_{C t}\right)$ given by Eq.(23) with $t_{c 2}\left(T G B_{C p}\right)$ calculated in [15]:

$$
t_{c 2}\left(T G B_{C p}\right) \simeq \sigma_{\perp}^{2}-1.06 \cdot\left(1+\sigma_{\|}\right)^{1 / 3}\left(16 \sigma_{\perp}^{2} \sigma_{\|}\right)^{1 / 3} b^{4 / 3}
$$


(We recalculated the numerical factor 1.06). The ratio of the upper critical fields for the $T G B_{C t}$ and $T G B_{C p}$ phases (at given temperature) is expressed as $\left(1+\sigma_{\|}\right)^{1 / 4}$, which is larger than one. This is consistent with Dozov's estimation when $\sigma_{\|} \gg 1$, but gives a more precise value of the numerical factor. Although the starting GL functional (Ti) is slightly different from that, used in 15 (see our remark after Eq.(困)), this does not change the final result: one can show that in a relevant limit, $\sigma_{\|} \gg \sigma_{\perp}$, expressions for $b_{c 2}\left(T G B_{C t}\right)$ calculated for both the functionals are the same. Note that standard perturbational analysis proves that the $T G B_{C t}$ and $T G B_{A}$ phases are stable with respect to small parallel tilting of the layers when angle $\varphi$ in Eq.(9) becomes nonzero.

To answer the second question, note that transition via an intermediate $T G B$ state is not preempted by the direct $N^{*}-S m$ transition when the upper critical temperature $t_{c 2}$ for the $T G B$ state is larger than the thermodynamical temperature $t_{c}$ of the direct transition. Recall first the calculations for the $T G B_{A}$ phase when $\sigma_{\perp}<0$ [1]. Comparison of $t_{c 2}$ given by Eq.(13) (without $\left(1+3 \sigma_{\|} / 4\right) b^{2}$ ) with the $N^{*}-S m A$ transition temperature $T_{N^{*} A}$ (6), which in dimensionless units is written as:

$$
t_{c}=\left(g K_{2}\right)^{1 / 2} b / D q_{0}^{3}
$$

gives the following criterion for stability of the $T G B_{A}$ phase:

$$
\kappa_{2}>1 / \sqrt{2} \quad \text { where } \quad \kappa_{2}=\left(g K_{2} / 2 \sigma_{\perp}^{2}\right)^{1 / 2} / 2 D q_{0}^{3}
$$

When $\sigma_{\perp}>0$ the $t_{c 2}$ transition temperature for $T G B_{C t}$ is given by Eq.(23), and the $N^{*}-S m C^{*}$ transition temperature $T_{N^{*} C^{*}}$ (6) in dimensionless units is written as:

$$
t_{c} \simeq \sigma_{\perp}^{2}-\frac{\left(g K_{2}\right)^{1 / 2}}{D q_{0}^{3}}\left(1-\sigma_{\perp} K_{2} / 2 K_{3}\right) b
$$

The criterion $t_{c 2}>t_{c}$ is always satisfied when $b$ is small enough, since at small $b, t_{c}$ is linear in $b$ and $t_{c 2}$ is proportional to $b^{4 / 3}$. So, strictly speaking, one can always obtain the $T G B_{C t}$ phase by preparation of the nearly racemic binary mixture of the left and right chiral molecules. The above consideration is valid only if $t_{c}(b)$ has a positive slope, that is, when $\sigma_{\perp}<2 K_{3} / K_{2}$. This condition was used in [15, 16] as a criterion for stability of the $T G B_{C}$ state. It is not very likely, however, to find a system where this condition will not be the case since in conventional $S m C^{*}$ liquid crystals $\sigma_{\perp}<1$ and $2 K_{3} / K_{2} \simeq 4-6$ [19]. In contrast, the practical identification of this small- $b T G B_{C t}$ phase can be quite difficult because of the very large cholesteric pitch. We formulate the realistic criterion for the existence of the $T G B_{C t}$ phase as a condition when it is stable in the vicinity of the critical point $M_{0}$. On the basis of the plot in Fig. 3c, one obtains that $t_{c 2}>t_{c}$ near $M_{0}$ if:

$$
\kappa_{2}>0.5 \sigma_{\|}^{1 / 4} \text {. }
$$

At realistic values of $\sigma_{\|} \simeq 10-100$ [24] this condition practically coincides with criterion for stability of the $T G B_{A}$ phase (31). The conditions (31) and (33) determine the position of the critical points $M_{A}$ and $M_{C}$ on the $N^{*}-S m A, C^{*}$ transition line of Fig.1 where the $T G B_{A}$ and $T G B_{C}$ phases first appear. 


\section{E. Resume}

We have revised the calculations of [15 for the $N^{*}-T G B_{A, C t}$ phase transition. The line of the upper critical temperature on phase diagram of Fig. 1 is reconstructed on the quantitative level and can be present in the relevant limit $\sigma_{\|}>1$ as follows:

On the left side of the point $M_{A}$ that is defined by condition (31), the direct $N^{*}-S m A$ first order transition takes place. The critical temperature of the transition is given by (30).

Between the points $M_{A}$ and $M_{0}$ the transition to the $T G B_{A}$ phase occurs. The point $M_{0}$ is placed at $0.36 \sigma_{\perp} \simeq \sigma_{\|}^{1 / 4} b$, inside the region $\sigma_{\perp}>0$. The upper critical temperature $t_{c 2}$ of the transition is given by (13) when $-\sigma_{\perp} / b>0.5+0.38 \sigma_{\|}$, and by (28) when $\sigma_{\perp} / b$ varies from a small negative value to $\sigma_{\perp} / b \simeq \sigma_{\|}^{1 / 4} / 0.36$. Both the asymptotics are matched in the intermediate region of negative $\sigma_{\perp} / b$.

In between the points $M_{0}$ and $M_{C}$ the sequence of transitions $N^{*}-T G B_{2 q}-T G B_{C t}$ takes place, the location of the point $M_{C}$ being given by the condition (33). The $T G B_{2 q}$ phase exists in a small temperature interval below $t_{c 2}$. It practically disappears on the right of the point $M_{1}$ where $\sigma_{\perp} / b>\sigma_{\|}^{1 / 4} / 0.19$. The upper critical temperature in between $M_{0}$ and $M_{C}$ is given by Eq. (23). The junction of $t_{c 2}$ transition lines for $T G B_{A}$ and $T G B_{2 q} / T G B_{C t}$ phases forms a kink in the tetracritical point $M_{0}$.

On the right side of the point $M_{C}$ the direct first order $N^{*}-S m C^{*}$ transition occurs at the critical temperature $t_{c}$, as is given by Eq.(32)

We have also calculated the width $\bar{l}_{b}$ of the $T G B$ slab at $T=T_{c 2}$ with respect to different parameters $b, \sigma_{\perp}, \sigma_{\|}$(Eqns. (17), (18), (24) and (27)). We summarize these results in Table I by the ratio $l_{b} / l_{d}=\bar{l}_{b} / \bar{l}_{d}=\bar{l}_{b}^{2} b / 2 \pi$, resulting from the topological constraint (II).

\section{ANALOGY WITH SPACE-MODULATED SUPERCONDUCTIVITY}

In this section we discuss a remarkable similarity between the $T G B$ state and the mixed (vortex state) in superconductors of type II noted first in [1]. We show that the $T G B_{C}$ phase corresponds to the mixed state in the "exotic" superconductors with space modulated order parameter.

Note first that, due to de Gennes [18,19] the $N-S m A$ transition is analogous to the superconducting phase transition because of similarity of the transformational properties of the order parameters: the space modulated function $\psi(r)=\psi_{0} e^{q_{0} \mathbf{n r}}$ in $S m A$ and the complex wave function $\Psi(r)$ in superconductor. The translation of $\psi(r)$ along the modulation vector $q_{0} \mathbf{n}$ (that is equivalent to multiplication of $\psi$ on a phase factor) corresponds to the gauge transformation in the superconductor.

The long range twist of $\mathbf{n}(r)$ in $N^{*}$ plays the role of the magnetic field destroying superconductivity. The $N^{*}-S m A$ transition via the intermediate $T G B_{A}$ phase occurs with continuous Meissner-like expulsion of the twist of $\mathbf{n}$. The rows of the screw dislocations resemble the Abrikosov vortex lattice.

The $S m C$ phase is characterized by the additional, transversal to $\mathbf{n}$ modulation of the order parameter resulted from the negative gradient terms in the CL model. Continuing the analogy with superconductivity one can tell that $N$-SmC transition corresponds to the transition to the superconducting state with nonuniform, space modulated order parameter. 
The $T G B_{C}$ phase therefore should be an analog of the mixed (Abrikosov) state of modulated superconductor, providing that magnetic field is perpendicular to the modulation.

On the quantitative level, the modulated superconductor in a magnetic field is described by the GL equation:

$$
t \psi+g|\Psi|^{2} \Psi=C_{\perp}(i \nabla-\mathbf{A})^{2} \psi+D(i \nabla-\mathbf{A})^{4} \psi
$$

where $\mathbf{A}$ is the vector potential of the field: $\mathbf{B}=\operatorname{curl} \mathbf{A}$. The modulation of the superconducting order parameter is provided by the gradient term with $C_{\perp}<0$. The upper critical field of this superconductor is calculated by neglecting the nonlinear term $g|\Psi|^{2} \Psi$ and solving the corresponding eigenproblem. It is easy to show that if $\mathbf{A}$ is chosen in the Landau gauge $(0, B x, 0)$, this procedure is exactly reduces to the case " $\sigma_{\|}=0$ " we considered in Sect. IIIB. According to calculations of Sect. IIIB, this superconductor should have the oscillating upper critical field, shown in Fig. 3a.

It is interesting to note that although the theory of space modulated superconductor was considered by Larkin and Ovchinnikov [20] and Fulde and Ferrel [21] more than thirty years ago, to our knowledge no direct experimental observation of this phase currently exists.

Note finally another interesting analogy between the $N^{*}-T G B_{A, C t}$ phase transition and the $B_{c 2}$ transition in "unconventional" multicomponent superconductor $U P t_{3}$ where the kinklike behavior of $B_{c 2}$ was observed [22]. The kink point in $B_{c 2}$ in $U \mathrm{Pt}_{3}$ is similar to the point $M_{0}$ in the $N^{*}-T G B_{A, C t}$ phase diagram: both points are provided by the intersection of eigenstates of the corresponding linearized GL equations.

\section{ACKNOWLEDGMENTS}

I am grateful to Monique Brunet who drew my attention to the problem and to Laurence Navailles and Philip Barois for fruitful discussions of the experimental aspects. This work was supported by the Brazilian Agency Fundacao de Amparo a Pesquisa em Minas Gerais (FAPEMIG) and by Russian Foundation of Fundamental Investigations (RFFI), Grant No. 960218431a. Part of the work was done during my stay in Université Montpellier II, France. 


\section{REFERENCES}

* E-mail: lukyanc@itp.ac.ru

[1] S. R. Renn and T. C. Lubensky, Phys. Rev. A38, 2132 (1988).

[2] J. W. Goodby, M. A. Waugh, S. M. Stein et al., Nature (London) 337, 449 (1989).

[3] J. W. Goodby, M. A. Waugh, S. M. Stein et al., J. Am. Chem. Soc. 111, 8119 (1989).

[4] G. Srajer, R. Pindak, M. A. Waugh, et al., Phys. Rev. Lett. 64, 1545 (1990).

[5] K. J. Ihn, J. A. N. Zasadzinski, R. Pindak, et al., Science 258, 275 (1992).

[6] L. Navailles, P. Barois and H. T. Nguyen, Phys.Rev. Lett. 71, 545 (1993).

[7] L. Navailles, R. Pindak, P. Barois and H. T. Nguyen, Phys. Rev. Lett., 42, 5224 (1995).

[8] L. Navailles, Ph.D. Thesis, L'Universite Bordeaux I (1994).

[9] H. T. Nguyen, A. Bouchata, L. Navailles, et al., J. Phys. II France 2, 1889, (1992).

[10] L. Navailles, H. T. Nguyen, P. Barois, et al., Liq. Cryst., 20, 653 (1996)..

[11] L. Navailles, C. W. Garland, J. Phys. II France, 6, 1243 (1996).

[12] A. Anakkar, A. Daoudi, J.-M. Buisine, et al., J. Therm. Anal., 41, 1501 (1994).

[13] A. Anakkar, A. Daoudi, J.-M. Buisine, et al., Liq. Cryst., 20, 411 (1996).

[14] T. C. Lubensky and S. R. Renn, Phys. Rev. A41, 4392 (1990).

[15] S. R. Renn, Phys. Rev. A45, 953 (1992).

[16] S. R. Renn and T. C. Lubensky, Mol. Cryst. Liq. Cryst. 209, 349 (1991).

[17] I. Dozov, Phys.Rev. Lett. 74, 4245 (1995).

[18] P. G. de Gennes, Solid State Commun. 14,997 (1972).

[19] P. G. de Gennes and J. Prost, The Physics of Liquid Crystalls (Oxford University Press, 1993).

[20] A. I. Larkin, Y. N. Ovchinnikov, JETP, 47,1136 (1964).

[21] P. Fulde and R. A. Ferrel, Phys. Rev. 135, 555 (1964).

[22] For a review see: I. Luk'yanchuk, M.Zhitomirsky, Superconductivity review, 1, 207, (1995).

[23] Jing-huei Chen and T. C. Lubensky, Phys. Rev. A14, 1202 (1976).

[24] L. J. Martinez-Miranda, A. R. Kortan, and R. J. Birgeneau, Phys. Rev. Lett. 56, 2264 (1986). 


\section{TABLES}

TABLE I. Location of the tetracritical point $M_{0}$ and the ratio $l_{b} / l_{d}$ in a different limit cases. Parameter $\epsilon$ varies from 0 when $K_{1,3} / K_{2}=0$ to 1.5 when $K_{1,3} / K_{2}$ is large.

\begin{tabular}{lcccc}
\hline \hline & \multicolumn{2}{c}{ Location of $M_{0}$} & \multicolumn{2}{c}{$l_{b} / l_{d}$ a } \\
& $b_{0}$ & $t_{0}$ & $T G B_{A}$, & $T G B_{2 q, C t}$, \\
& & & $b>b_{0}$ & $b<b_{0}$ \\
\hline$\sigma_{\|} \ll 1$ & $0.5 \sigma_{\perp}$ & $0.75 \sigma_{\perp}^{2}$ & $0.8 \varepsilon$ & $\sigma_{\perp} / 2 \pi b$ \\
$\sigma_{\|} \gg 1$ & $0.36 \sigma_{\perp} / \sigma_{\|}^{1 / 4}$ & $0.43 \sigma_{\perp}^{2}$ & $0.7 \varepsilon \sigma_{\|}^{-1 / 4}$ & $\varepsilon\left(4 \sigma_{\perp} / b \sigma_{\|}\right)^{1 / 3}$ \\
\hline \hline
\end{tabular}

aThe ratio $l_{b} / l_{d}$ is given at $N^{*}-T G B$ transition line. At lower temperatures $l_{b} / l_{d}$ increases rapidly. 
FIGURES 


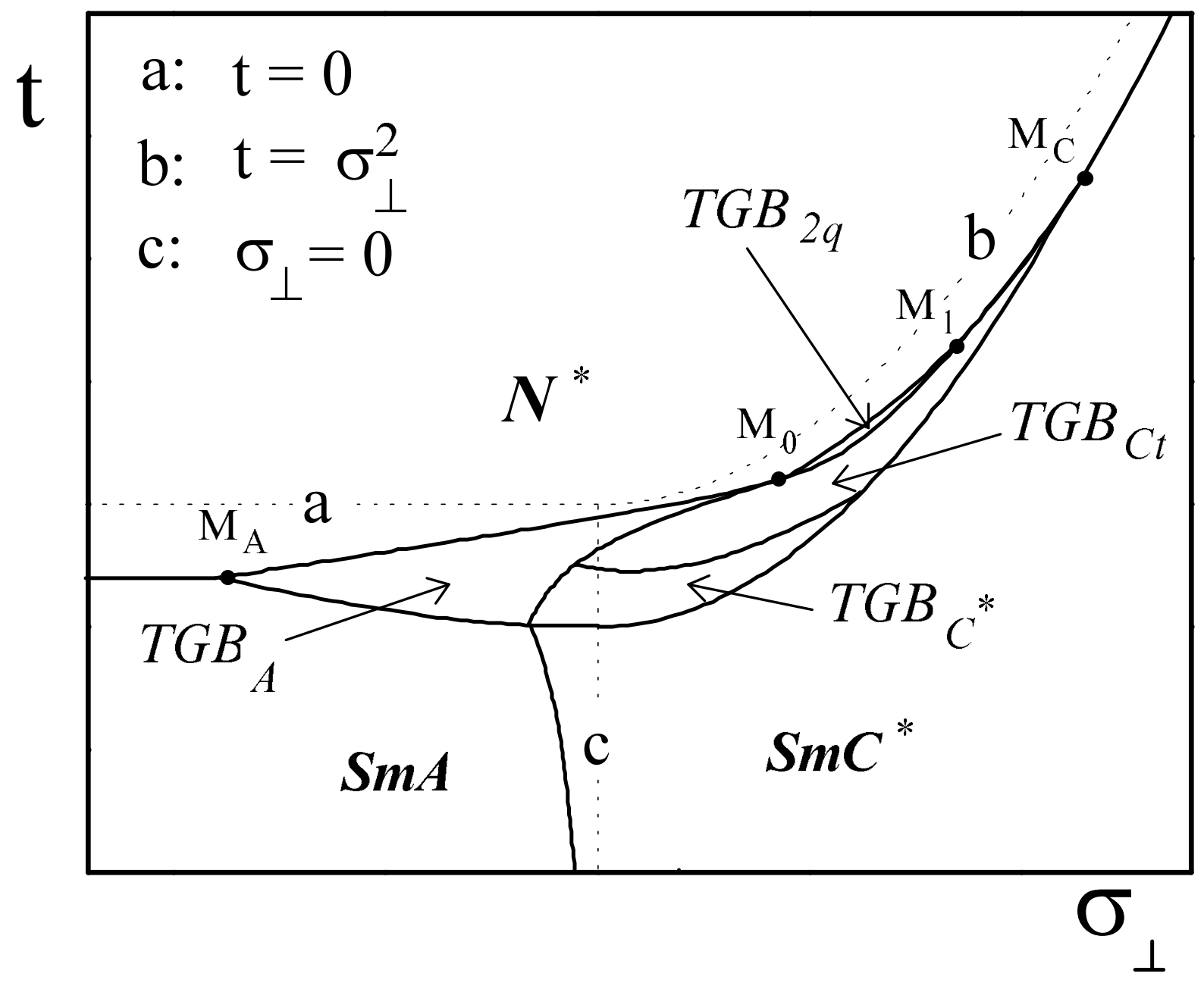


FIG. 1. The phase diagram of $T G B$ phases. The model parameters $t, \sigma_{\perp}$ are controlled by the experimental conditions. We predict a new $T G B_{2 q}$ phase and penetration of the $T G B_{A}$ phase in the $S m C^{*}$ region where $\sigma_{\perp}>0$. Dotted lines a, b and c present the $N-S m A-S m C$ diagram in the nonchiral case. 

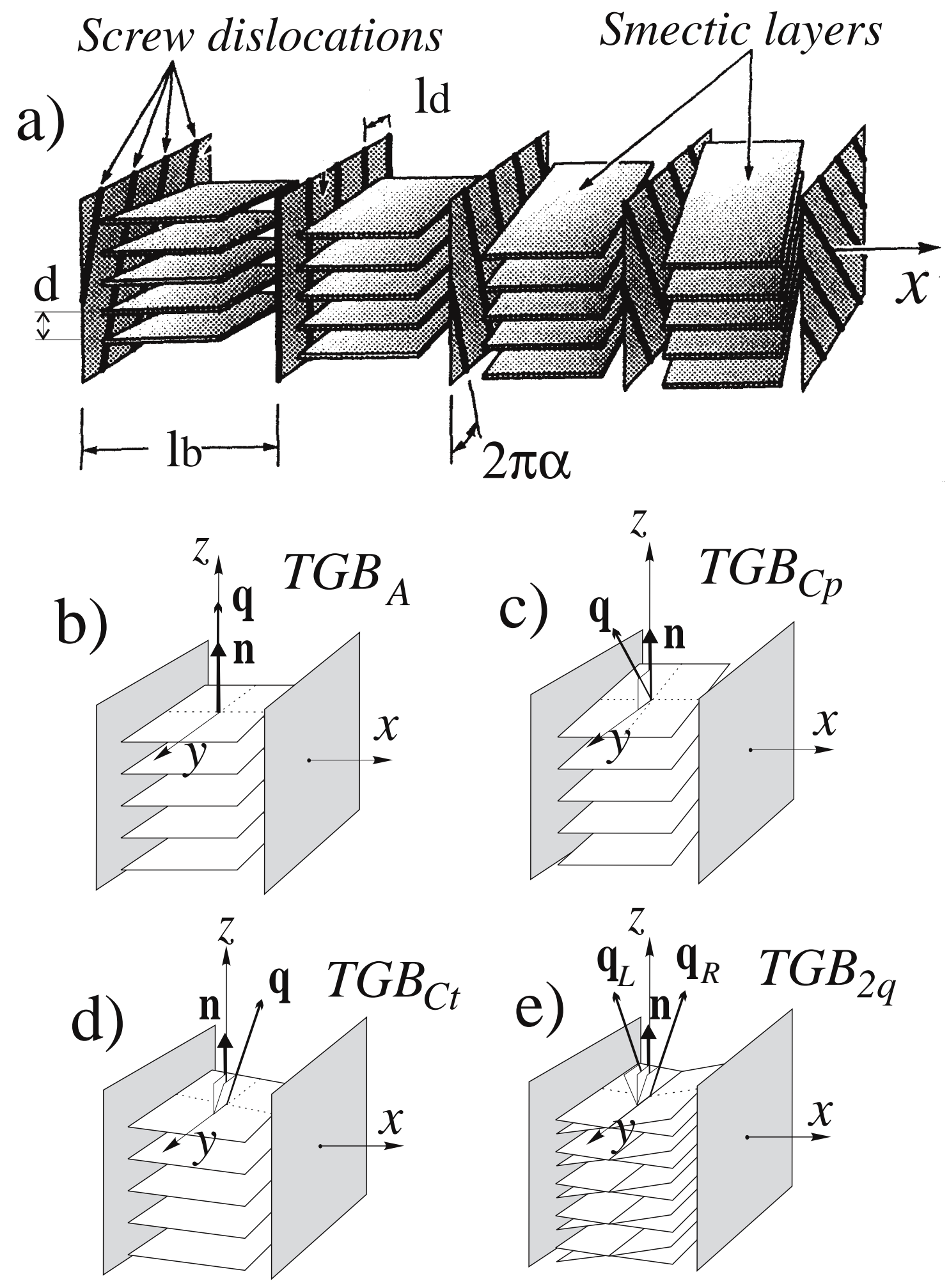

FIG. 2. Structure of different TGB phases. 

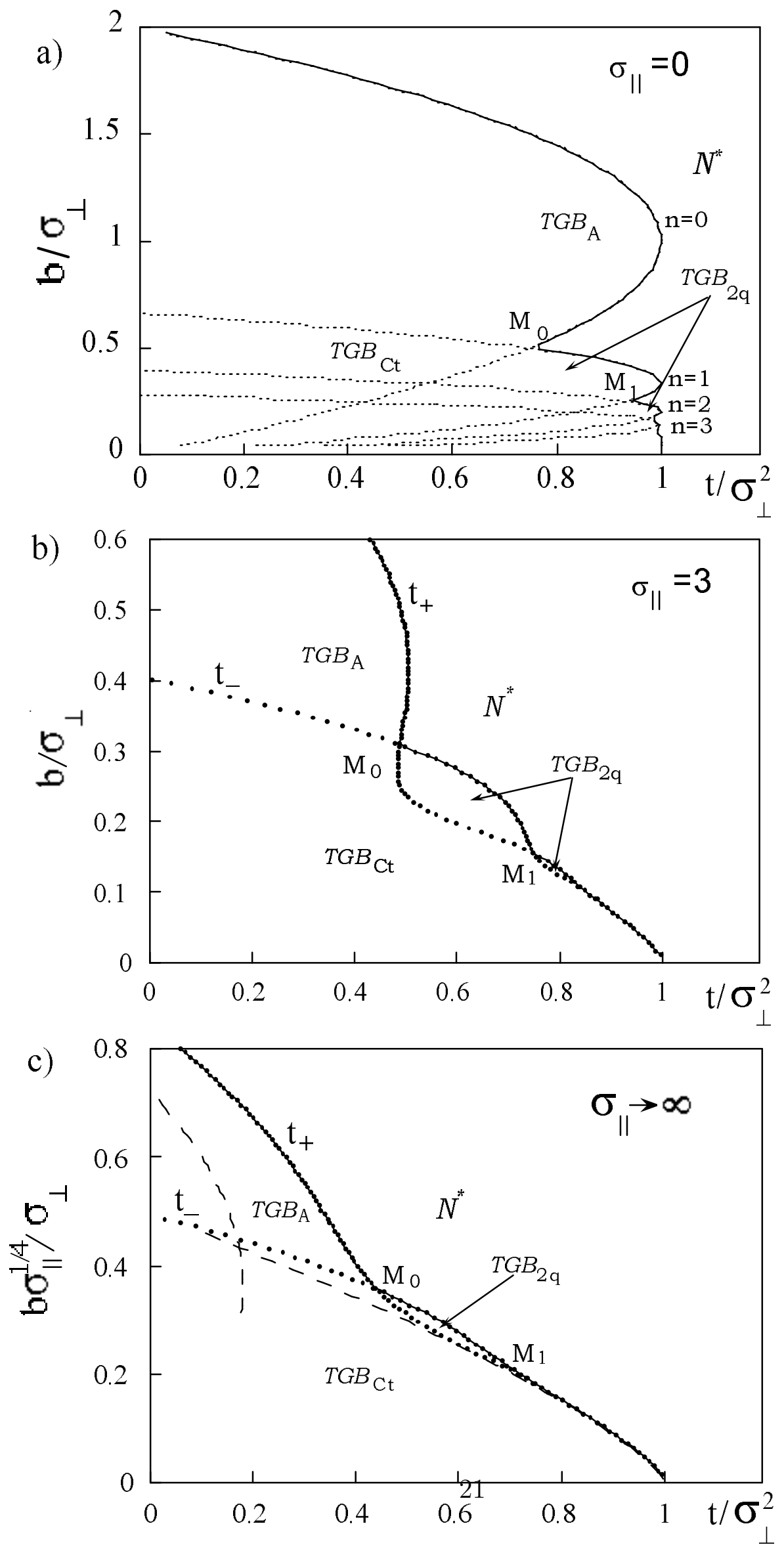
FIG. 3. Oscillating behavior of the lowest eigenstates of the lineariezed CL model that correspond the Cholesteric $\left(N^{*}\right)-T G B_{C t}$ transition as a function of the model parameters: $t / \sigma_{\perp}^{2}$, $b / \sigma_{\perp}$ and $\sigma_{\|}$. The concurrence between two nearly degenerate lowest eigenlevels (the minimal one corresponds to $t_{c 2}$ ) leads to spliting of the transition by appearance of either the $T G B_{A}$ phase or the intermediate $T G B_{2 q}$ phase. Dashed lines in (c) present the asymptotics calculated in the text. 